\title{
Selected structural elements of the wing to increase the lift force
}

JEL: L93 DOI: 10.24136/atest.2018.494

Data zgłoszenia: 19.11.2018 Data akceptacji: 15.12.2018

The article presents a currently used structural elements to increase the lift force. Presented mechanical and no-mechanical construction elements that increase the lifting force. The author's attention to the new direction of flow control using a DBD plasma actuator. This is a new direction of active flow control.

Słowa kluczowe: aerodynamic, high-lift device, plasma actuator DBD

\section{Introduction}

One of the many causes of accidents and disasters in aviation is a loss of lift force during flight, most often caused by flow disturbances around a wing profile. This is especially true when starting or landing, the wing operates in the limiting angles of attack. There may be a stall and catastrophic disturbance of the flight path.

Wing profiles have a specific geometry that determines the use of a particular profile in aircraft constructions. The parameters which influence the choice of the design of the profile are; speed of flight, wing loading, purpose aircraft. Each airfoil has determined experimentally or by calculation the maximum angle of attack a and the minimum speed at which no loss of aerodynamic lift. Increasing the angle of attack a moves the boundary layer separation point towards the leading edge, which leads to a turbulent air flow along the chord of the wing profile. The phenomenon is accompanied by breaking the air stream from the surface of the wing, causing a loss of lift force, which often lead to disaster.

The article discusses currently applied construction solutions improving the aerodynamic properties of the airfoil which increase flight safety. Also described new solutions allows to control of the boundary layer using a plasma actuator DBD.

\section{A review of selected construction solutions} that improve lift force

1.1. Non-mechanical structural elements of the wing improving the lift force

Airplanes are equipped with design solutions that allow improving the aerodynamic properties. The main design solution, which affects the safety of the flight is to select the right wing profile, adapted to type of aircraft e.g. load, speed, range. There are several types of airline profiles depending on the applications; classic, laminar, supercritical airfoil, supersonic. Due to the symmetry of the profile, we stand out; symmetrical, semi-symmetrical, flat bottom, under-cambered, Illustrative profiles are shown in Figure 1. The choice of airfoil depends on the application and purpose. The symmetrical profile does not generate lift force at zero angle of attack, therefore it is most often used with a vertical or horizontal stabilizer. Profiles for aircraft flying at supersonic speeds are of a small thickness. The choice of profile depends on the use of the aircraft. Profile selection is made in the design phase, depends on many factors and, above all, on the purpose of the aircraft. a)

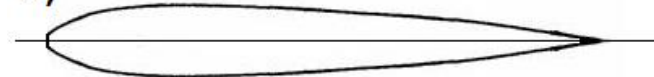

b)

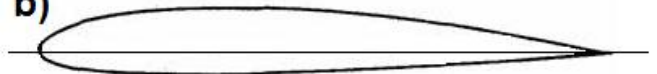

c)

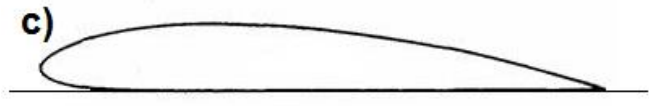

d)

Fig. 1. The most common airline profiles; a) symmetrical, b) semisymmetrical, c) flat bottomed, d) under-cambered

Even a properly selected wing profile in certain conditions (at high angles of attack) loses the lift force. To counteract this, construction offices introduce elements that improve the flow laminarity and improve safety.

\section{Wing cuff}

Wing cuff is a static aerodynamic modification of the front part of the wing, which is a downwardly curved nose of the profile to improve / soften the stall characteristics. Modification is subjected from $50 \%$ to $70 \%$ of the outer length of the wing, which makes the outer part of the wing cuff, has a lower angle of attack and continues to fly even when the inner section without the cuff loses the lift. The wing section with a wing cuff has an increased rake angle, as shown in Figure 2.

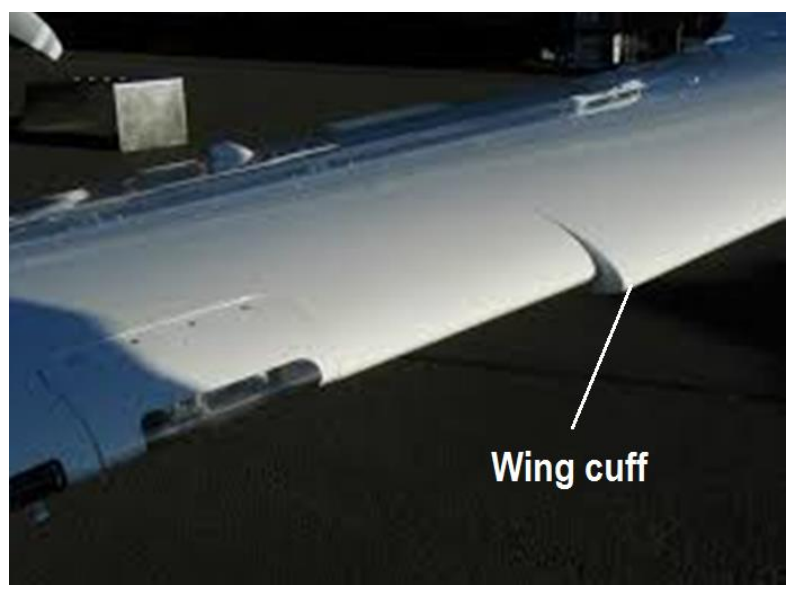

Fig. 2. Wing with marked wing cuff

Another advantage using wing cuff lowering the stall speed and improving the effectiveness of ailerons / flaps at large angles of attack [1].

\section{Wing fence}

Engineers are introducing new design solutions to improve the aerodynamics of the aircraft. The changes introduced by the construction offices in the construction of the airfoil allow the improvement of aerodynamic properties. Elements that improve the stability 
of the flight, which are placed directly on the surface of the wing on its upper surface along the profile (parallel to the plane of the fuselage) are wing fence. The wing fence is a plate perpendicular to the wing plating usually on its upper surface. Most often there are two versions; the first one in which the plate is placed over the entire width of the wing (chord) is the long fence version and the second version in which the plate is only on the front part of the wing is a short fence as shown in figure 3 . The action of the wing fence is based on the fact that the air streams moving over the swept wing tend to move towards the wing tips. The use of the wing fence causes the air stream to encounter an obstacle in the form of a vertical plate that directs the air stream back to the surface of the wing. Placing the wing fence on the wing delays or eliminates this effect, preventing too much flow along the wing [2]. The consequence of not parallel flow of the air stream is the disturbance of the transverse plane balance. The use of the wing fence improves the transverse stability of the aircraft.

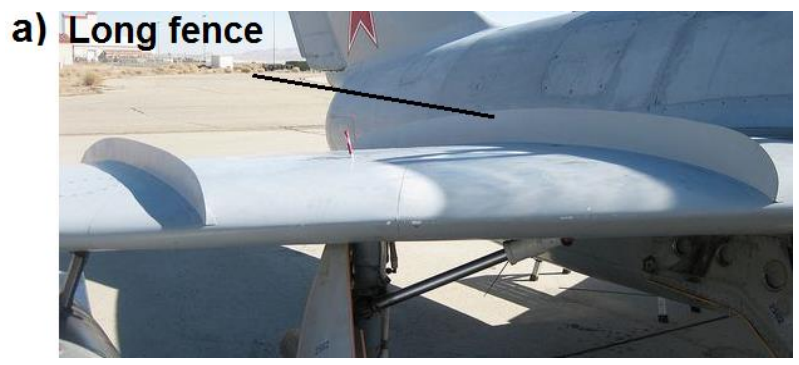

b)

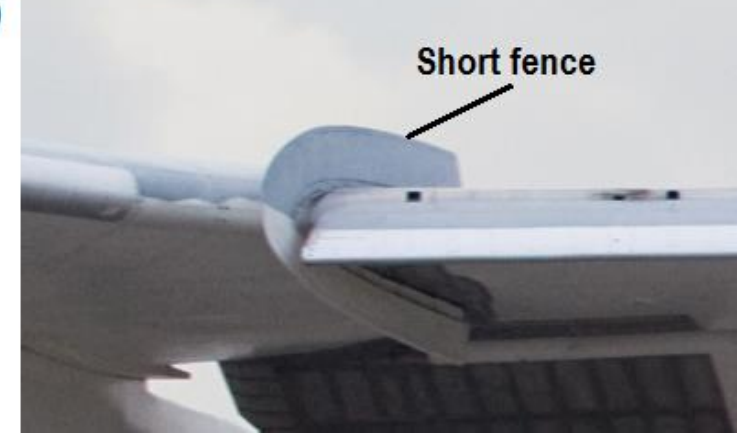

Fig. 3. Wing fence; a) long fence MiG-15, b) short fence Tu-22

\section{Vortilons}

Other non-mechanical elements improving the aerodynamic properties placed permanently on the underside of the wing are Vortilons.. The name Vortilons comes from the words VORTEX and pYLON. Vortilons consist of vertical tiles placed parallel to the direction of flight (perpendicular to the surface of the wing) mounted close to the leading edge from the bottom as shown in Figure 3 on the example of the aircraft wing Embraer erj 145.

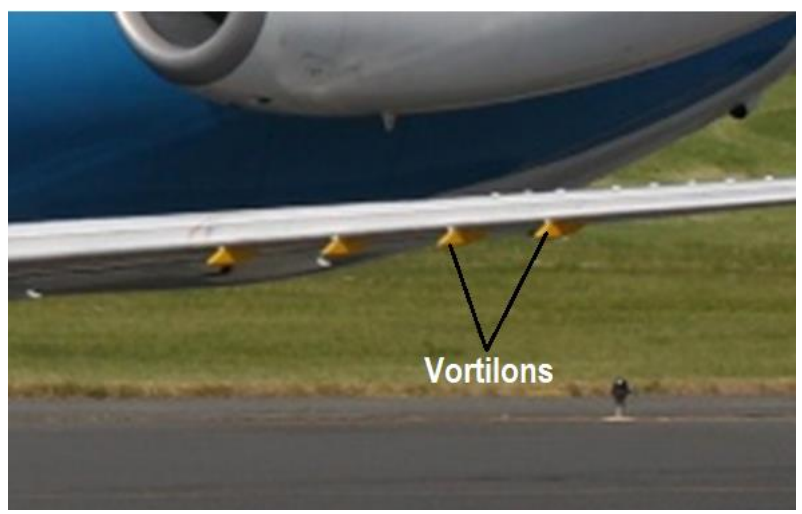

Fig. 3. Vortilons on the lower part of the wing, Embraer erj 145
The Vortilons aerodynamic system has an important function, similar to the Vortex generator, but do not put as much resistance as the Vortex generator. The main function of Vortilons is to generate a whirlwind over the top of the plane only at a high attack angle. The operation of the Vortilons system also has a positive effect on stalling, and also increases the efficiency of the ailerons.

\section{Vortex generator}

Vortex generator are aerodynamic devices similar to Vortilons. In contrast to the Vortilons, the Vortex systems are placed on the upper surface of the wing. The Vortex generator looks similar to the a small fin placed on the upper surface of the wing near the upper leading edge. They are placed at a small angle alternately in sections as shown in Figure 4. The Vortex generator is used to improve the flow of the wing or other aircraft parts (ailerons or control surfaces), by creating a vortex in the boundary layer, thereby preventing or delaying its separation.

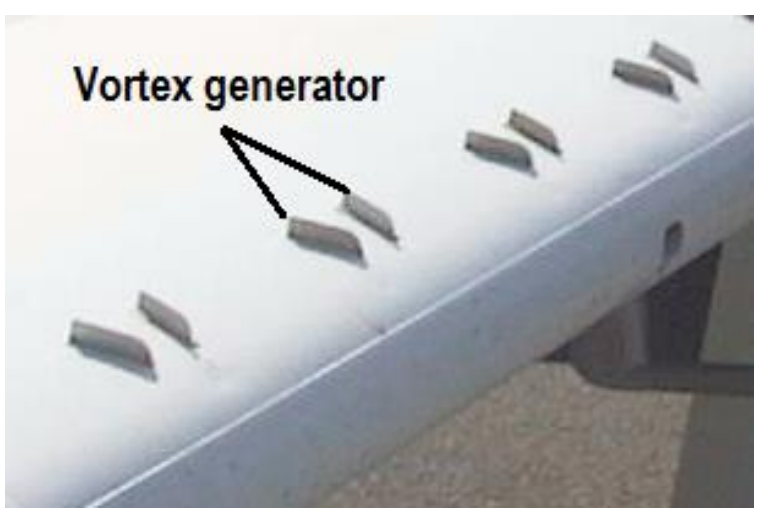

Fig. 4. Vortex generator placed on the leading edge of the wing

\section{Winglet}

Winglets are aerodynamic elements placed permanently on the tip of the wings, having the shape of the wing / fin. Winglets are used primarily to reduce induced drag associated with the formation of a vortex path coming down from the tip of the wing. The use of winglet elements improves the aerodynamic of the wing and reduces fuel consumption, which increases the range of the aircraft, and flight is more comfortable, because it helps to dampen vibrations, also improves the airplane's performance allowing for shorter takeoff. Another advantage of using winglet is a significant reduction in the intensity of wake vortices, these whirls are a threat to airplanes flying behind such air disturbances. The shape of the winglet is dependent on the aerodynamic properties of the wings and the aircraft type. Exemplary shapes are shown in Figure 5.

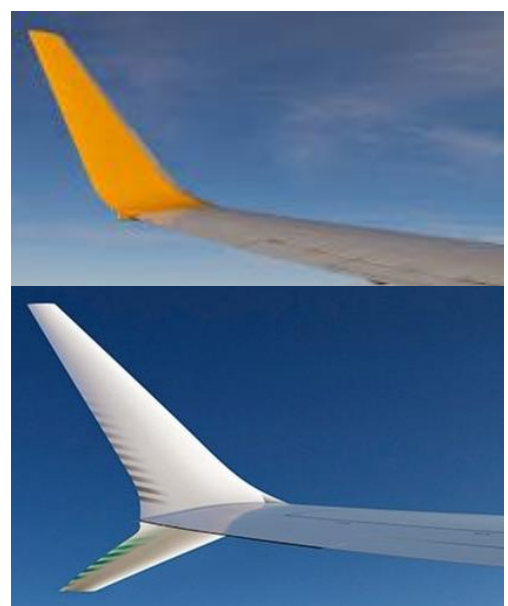

Fig. 5. Selected winglets 


\subsection{Mechanical structural elements of the wing influencing} the lift force

In previous chapters 1.1, non-mechanical elements are described to improve aerodynamic and flight safety. In civil and military airplanes, mechanical solutions are used to improve flight safety and stability. Chapter 1.2 presents selected elements of wing mechanization, which increase the lift and flight safety. Wing mechanization involves the use external elements to increase the lift or aerodynamic resistance (spoilers and aerodynamic brakes). These devices are mainly used during take-off and landing and landing approaches, when the wing is operated at around border angles of attack These systems change the flow of the wing by changing the shape of its profile, increasing the surface or changing flow the boundary layer in a place where the flow can be torn off.

\section{Slats}

The mechanical elements of the wing are used to prevent separation air stream from the surface of the wing, which leads to loss of lift.. An element that prevents the air stream from detaching itself are the slats. Action slats begins when the wing reaches the approach angle close to the stall. Slats are one of the elements of wing mechanization, which are placed along the leading edge of the wing. Slats are designed to improve the aerodynamic properties of the aircraft during flight at low speeds and high angles of attack, by delaying the detachment of air streams. The principle of slats operation is shown in Figure 6.

a)
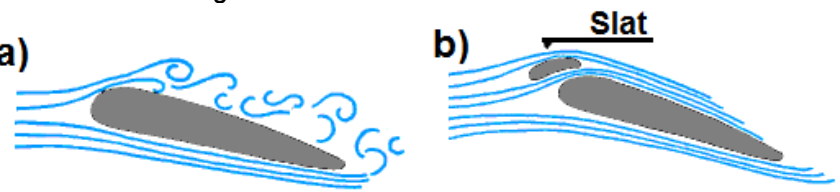

Fig. 6. The principle of operation slats

The most common construction is the mobile slat presented in Figure $7 \mathrm{~b}$ and the fixed slat Figure $7 \mathrm{a}$.

a)

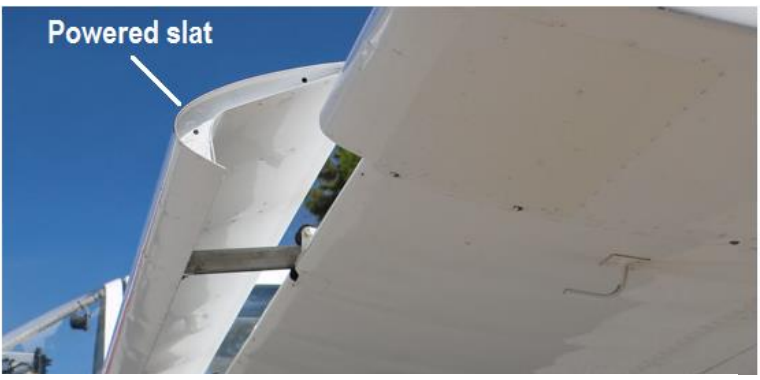

b)

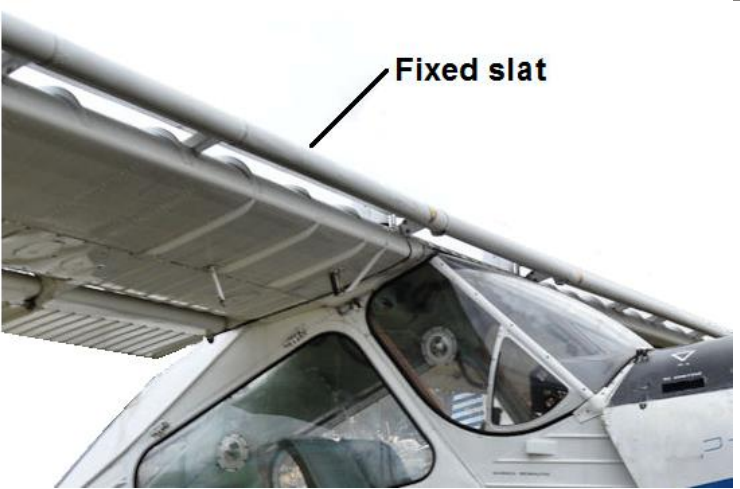

Fig. 7. Slats; a) powered slat, b) fixed slat.

Flaps

The flap is an element in the rear part of the wing of the aircraft, it allows for a significant increase in lift, especially during the take-off and landing of the aircraft. There are several types of flaps depending on their construction, which allow to increase the lifting force, the most commonly used flap construction in passenger planes are triple slotted flaps. The principle of triple slotted flaps is shown in Figure 8.

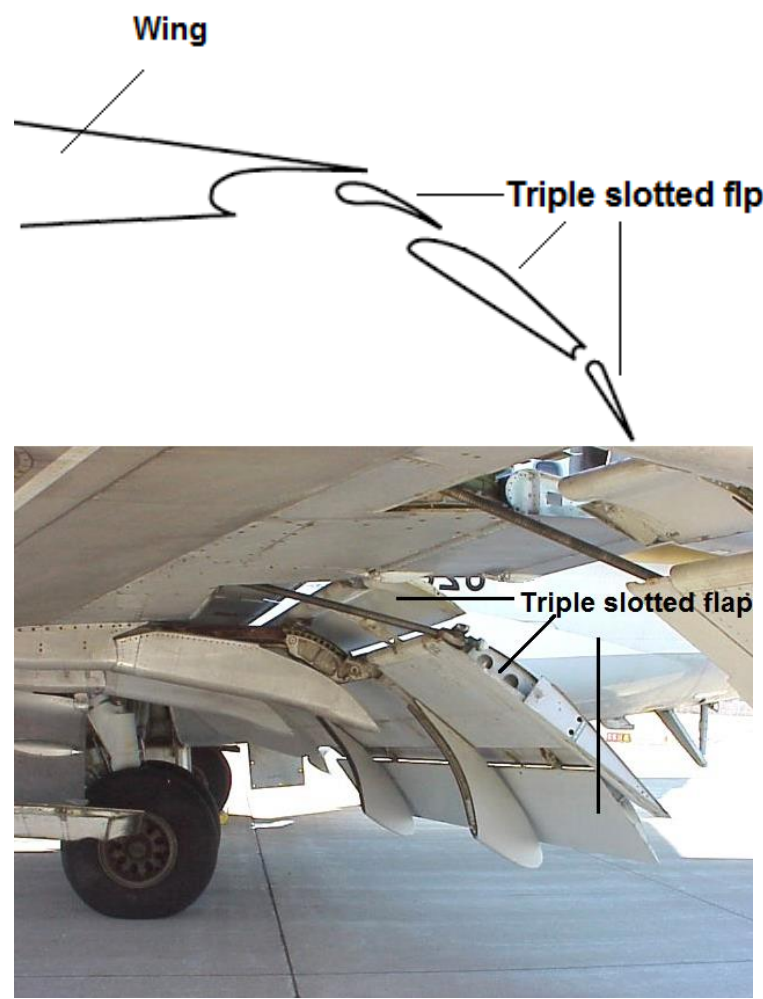

Fig. 8. The construction of triple slotted flaps

This solution allows to increase the surface of the wings and the curvature of the profile camber line. Triple slotted flaps hatches have a very complicated release mechanism, which is subjected to high aerodynamic loads during take-off and landing

\section{Krueger flap}

The Krueger flap is an aerodynamic mechanism located in the front part of the wing (leading edge), a small part of the lower wing covering rotates down perpendicular to the direction of flight (parallel to the leading edge). At the time of use, this flap rotates forward, increasing the airfoil area near the leading edge. Krueger flap construction shown in Figure 9.

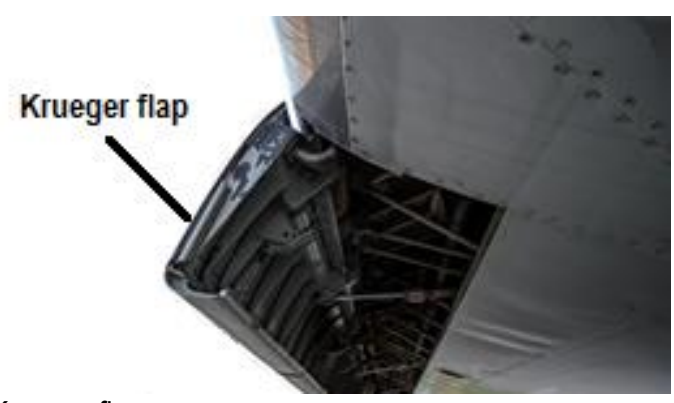

Fig. 9. Krueger flap.

The Krueger flap is mainly used during takeoff and landing (landing approach), it increases the lifting force at large rake angles and low speed, by changing the shape of the profile. 
2. The use of plasma actuator DBD for active control boundary layer flow

A new solution currently undergoing tests for active flow control using plasma technologies DBD (Dielectric Barrier Discharge) barrier discharges [7]. The DBD system does not have any moving parts such as lines, valves, diaphragms, cylinders or gears, in order to activate it, which does not create design problems affecting the construction of the aircraftConstruction of the system is simple, and consists of two flat electrodes separated by a dielectric, the most common configuration of the DBD is asymmetrical circuit shown in Figure 10a. One of the electrodes is exposed to the direct action of the flowing air, second electrode is fully covered with a dielectric material. The use of a dielectric prevents the arcing or sparks discharge that cause a local increase in temperature. The electrodes are connected to a supply of high voltage (several kV). The DBD system built in this way is mounted directly on the surface of the wing (figure 10b). When the electrodes are supplied with high voltage, the ionized air (plasma) moves from exposed electrode towards the electrode covered with a dielectric. As a consequence of the elastic collisions between migrating molecules of charged and inert gas particles, the neutral ones increase their rate, causing the "electric wind" which takes place in close proximity to the surface [6]. The operation of the actuator DBD, depends on many factors; dielectric type, supply voltage, air flow speed, electrode geometry and operating frequency of the power supply system [3-5]. Plasma actuators have many clear advantages over other flow control devices.

a)
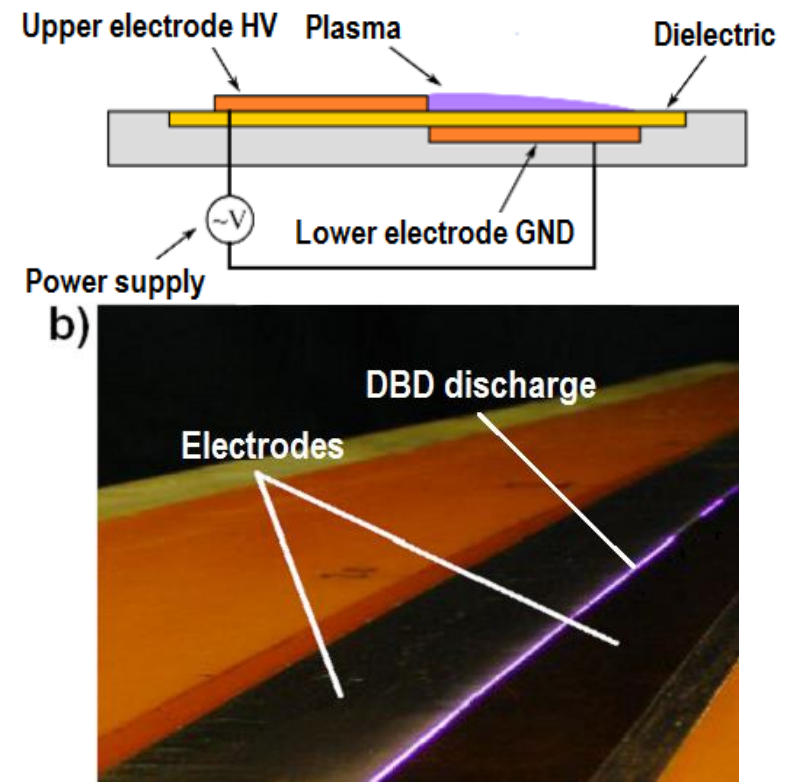

Fig.10. Construction and operation; asymmetric DBD plasma actuator $a)$, the actual plasma actuator DBD model placed on the wing $b$ )

The main advantages of using DBD plasma actuators for flow control are:

- Plasma actuators are fully electronic without moving parts,

- They can withstand high loads,

- They are not required slots or recesses,

- Plasma actuators can work in both constant and unstable modes,

- Plasma actuators have a large bandwidth,so that they have a fast response to feedback control.

- They are thin (small drag)

\section{Summary}

The article presents selected elements of wing construction that improve aerodynamic and flight safety. The first chapter presents selected non-mechanical elements that improve the lift capacity currently used in aviation. Operation of these elements is focused on stabilizing or gluing to the flow boundary layer. This task is performed by the reduction / "gluing" boundary layer or by introduction of controlled disorders (vortices) enabling to increase energy of the boundary layerThe next subchapter presents selected elements of wing mechanization that improve aerodynamic properties of the wing and flight safety. The use of wing mechanization allow to increase the lifting force by sliding out slat and flap, which allow to increase the wing surface and change the wing chord. The second section describes a new solution which discharges DBD to actively flow control the boundary layer, they are currently being tested in many research centers. This solution is a relatively new way of actively controlling the boundary layer, requires many tests to be carried out before being implemented for common use in communication airplanes.

\section{Reference:}

1. Crane, Dale: Dictionary of Aeronautical Terms, third edition, page 144. Aviation Supplies \& Academics, 1997.

2. Satran W., Johnson D., Effects of Wing-Leading-Edge Modifications on a Full-Scale, Low-Wing General Aviation Airplane. NASA TP-2011, 1982

3. Gnapowski E., Gnapowski S., Impact Porous Dielectric Silica Gel for Operating Voltage and Power Discharge Reactor, World Academy of Science, Engineering and Technology International Journal of Electrical, Electronic Science and Engineering, Vol:8 No:2, 2014.

4. Gnapowski E., Gnapowski S., Pytka J., Effect of Mesh Geometry on Power, Efficiency and Homogeneity of Barrier Discharges in the Presence of Glass Dielectric, IEEE Transactions on Plasma Science, 2018, Vol. 46: p-p 3493 - 3498.

5. Gnapowski E., Gnapowski S., Pytka J., The impact of dielectrics on the electricalcapacity, concentration, efficiency ozonegeneration for the plasma reactor with meshelectrodes, Plasma Science and Technology, Vol. 20, Nr 8, p-p 1-7

6. Seraudie A., Vermeersch O., and Arnal D., "DBD Plasma actuator effect on a 2D model laminar boundary layer. Transition delay under ionic wind effect.", 29th AIAA Applied Aerodynamics Conference, Fluid Dynamics and Co-located Conferences.

7. Seraudie A., Aubert E., Naudé N., and Cambronne J., "Effect of Plasma Actuators on a Flat Plate Laminar Boundary Layer in Subsonic Conditions", 3rd AIAA Flow Control Conference, Fluid Dynamics and Co-located Conferences.

\section{Selected structural elements of the wing to increase the lift force}

The article presents a currently used structural elements to increase the lift force. Presented mechanical and no-mechanical construction elements that increase the lifting force. The author's attention to the new direction of flow control using a DBD plasma actuator. This is a new direction of active flow control.

Keywords: aerodynamic, high-lift device, plasma actuator DBD

Autorzy:

dr Ernest Gnapowski - Department of Transport, University College of Enterprise and Administration, Lublin, egnapowski@gmail.com 